\title{
Study of Jujube Polysaccharied on Protection from Lipid Peroxidation Damage of Cardiac Muscle Tissue after Exercise
}

\author{
Li Liu \\ Sports Department, Northwest University, Xi'an 710069, China \\ 35739659@qq.com
}

Keywords: Jujube polysaccharide; Intensive exercise; Cardiac muscle; Lipid peroxidation

\begin{abstract}
By the method of experimental animals, to examine the jujube polysaccharide on protection from lipid peroxidation damage of cardiac muscle tissue in exercise rat . Twenty-four SD mice were selected and divided into control group, exercise group and exercise + jujube polysaccharide group randomly. There were eight mice in each group. Mice of exercise group were trained on intensive treadmill for eight weeks. Test rat cardiac muscle tissue antioxidant enzyme activity and serum GOT activity index. The result should that levels of cardiac muscle MDA increased significantly in rats after intensive exercise; while those of SOD, GSH-Px, CAT and Serum GOT decreased significantly in rats after intensive exercise. The supplement of jujube polysaccharide degraded levels of cardiac muscle MDA after intensive exercise; while raised those of SOD, GSH-Px, CAT and Serum GOT after intensive exercise. The jujube polysaccharide could increase the activity of antioxidase, relieve the peroxidation damage to cardiac muscle caused by maximal intensity endurance training.
\end{abstract}

\section{Introduction}

Red dates polysaccharide is an important kind of physiological activator. It is classified as a kind of high molecular polymer polymerized by monosaccharides. Many medical researches indicates that red dates polysaccharides can clear the free radicals and prevent lipa from oxidation[1]. It can also improve the activity of superoxide dismutases (SOD) and catalase (CAT) in mice's blood, liver and brain tissues; lower the concentration of malonaldehyde (MDA) in mice's blood, liver and brain tissues[2]. Our research builds a model of High-intensity endurance exercise training of mice to measure the activity of serum enzyme and the free radical metabolism of mice's heart tissues. Also, we will discuss the possibility and mechanism of how the dates benefit the Exercise-induced cardiac injury, thus offering experimental data for the further development and application.

\section{Materials and Methods}

Experimental Subject. 24 male SD mice, weighing from 220-280( offered by the Experimental Animal Center of the Medicine College of Xi'an Jiaotong University), animal feed purchased simultaneously. The experiment begins after one week's feeding.

Experimental Design. Divide the mice into A, B, C, three groups at random and each group is made up of eight mice. Group A is the sedentary control group, Group B is the exercise control group and Group is the exercise and polysaccharide group. Each group will be kept in separated cages under the temperature of $20 \pm 2^{\circ} \mathrm{C}$, humidity of $44 \%-55 \%$, 0 slope, good ventilation and natural light. Group $\mathrm{A}$ is kept in the sedentary group while Group B and $\mathrm{C}$ will be trained on the animal treadmill for eight weeks. During the first five weeks, the incremental pressure training will be given to each group, six days per week, 25 minutes per day, the running speed will increase progressively at $15 \mathrm{~m} / \mathrm{min}, 22$ $\mathrm{m} / \mathrm{min}, 27 \mathrm{~m} / \mathrm{min}, 31 \mathrm{~m} / \mathrm{min}$ and $35 \mathrm{~m} / \mathrm{min}$. During the last three weeks, high intensity training will be given to each group, seven days per week, 35 minutes per day, 0 slope and the running speed is 35 $\mathrm{m} / \mathrm{min}$. Each group can feed and drink freely everyday. At the same time, Group $\mathrm{C}$ will be fed with red 
dates polysaccharide at the amount of $50 \mathrm{mg} / \mathrm{kg}$ per day( the red dates are purchased from Zhejiang Dashanhe Mushroom Co., Ltd). An equal volume of saline will be given to Group A and B.

Sampling Methods. 24 hours later after the mice finished its last training, decapitate the mice to gain the hard tissues and myocardial tissues. Put 3-4ml hard tissues into anticoagulation centrifuge tubes and then cryopreserve the supernatant. Make homogenate out of $200 \mathrm{mg}$ myocardial tissues and centrifuge to gain the supernatant for spare.

Target Test. The SOD determination uses the xanthine oxidase method; the MDA determination uses the TBA method; the GSH-Px determination uses the DTNB method; the CAT determination uses the ultraviolet spectrophotometry; the GOT determination uses the Method for determination of Lai's colorimetry. ( all the kits are purchased from Nanjing Jiancheng biological engineering institute)

Statistical Analysis. Using SPSS 18.0 software processing experimental data, the results to $\mathrm{X} \pm$ SD expressed in T-test for statistical analysis.

\section{Experimental Result}

The results are shown in Table 1. The serum GOT activity of Group A and Group B are higher than Group $\mathrm{C}$, and both showed significant differences $(\mathrm{P}<0.05)$; Group A is slightly higher than group $\mathrm{C}$ group, but there' s no significant difference (P>0.05); Compared with Group A, the SOD, GSH-Px, CAT activity of Group C decreased significantly; compared with Group B, the SOD, GSH-Px, CAT activity of Group $\mathrm{C}$ increased significantly $(\mathrm{P}<0.05)$; compared with Group A, the SOD, GSH-Px activity of Group $C$ showed significant difference. Compared with Group A, the CAT activity of Group $\mathrm{C}$ showed no significant difference. The MDA content trends are: A group $<$ Group $\mathrm{C}<\mathrm{Group}$ B. The MDA content is significantly higher in Group B than that in Group A and Group C, which means there exists a significant difference $(P<0.05)$, indicating that the jujube polysaccharide can effectively reduce the damage caused by high-intensity exercise on the heart.

Table1 Effect of Jujube Polysaccharide on the Activity of Antioxidase and the Content of MDA in the Cardiac Muscle of the Training and serum GOT active

\begin{tabular}{llll}
\hline & $\mathrm{A}$ & $\mathrm{B}$ & $\mathrm{C}$ \\
\hline CAT & $1.12 \pm 0.09$ & $0.72 \pm 0.14^{* *}$ & $1.14 \pm 0.53^{\Delta}$ \\
GSH-Px & $135.36 \pm 6.94$ & $113.01 \pm 6.14^{* *}$ & $159.85 \pm 17.92^{* \Delta \Delta}$ \\
SOD & $101.07 \pm 8.31$ & $86.91 \pm 10.04^{* *}$ & $134.78 \pm 9.60^{* \Delta \Delta}$ \\
MDA & $2.90 \pm 0.54$ & $4.81 \pm 0.76^{* *}$ & $1.09 \pm 0.59^{* \Delta \Delta}$ \\
GOT ( & $45.06 \pm 4.01$ & $62.00 \pm 3.07^{*}$ & $47.12 \pm 1.39^{\Delta}$ \\
\hline
\end{tabular}

Note: * indicates a significant difference $(\mathrm{P}<0.05)$ in group $\mathrm{A}$; ** denotes a highly significant difference from Group $\mathrm{A}(\mathrm{P}<0.01) ; \Delta$ indicates a significant difference from $\operatorname{Group} \mathrm{B}(\mathrm{P}<0.05) ; \Delta \Delta$ said there was a significant difference from Group $\mathrm{B}(\mathrm{P}<0.01)$.

\section{Discussion and Analysis}

Lipid peroxidation is a series of free radical reaction occurs at the unsaturated fatty acids covalently. Under normal physiological conditions, due to complete existence of the anti-oxidative enzyme systems(CAT, SOD, GSH-Px) and natural antioxidants (coenzyme Q10, VitC, VitE, VitA, glutathione) in the body, the oxygen radicals are promptly removed, the free radicals are at a low concentration harmless state of dynamic equilibrium. Under conditions of high intensity or exhaustive exercise, the oxygen free radicals will increase sharply. The body cannot rely on its own antioxidant system to remove excess free radicals effectively. So the lipid peroxidation increases, the attack on the cell membrane, resulting in cell structure and function decline, thereby causing tissue damage, which is an important cause of exercise-induced fatigue generated[3]. Heart, also called the "blood pump", is the driving organs of blood circulation. The heart working condition will directly affects the transportation of nutrients and metabolic waste transportation. When doing high-intensity or exhaustive exercise, the body's energy consumption increased and metabolic process enhanced. If cardiac tissues are damaged, circulatory system disorders, exercise capacity will be significantly decreased. 
The experimental results show that after prolonged endurance training myocardial tissue SOD, GSH-Px, CAT activity decreased significantly, MDA levels were significantly increased $(\mathrm{P}<0.01$ or $\mathrm{P}<0.05)$. By taking jujube polysaccharide, heart tissue SOD, GSH - Px, CAT activity are improved and MDA levels decreased significantly ( $\mathrm{P}<0.01$ or $\mathrm{P}<0.05$ ). Superoxide dismutase (SOD) is the first body defense line of removing free radicals, whosefunction is to remove the superoxide anion radical (· O2-) and disproportionate it to $\mathrm{O} 2$ and $\mathrm{H} 2 \mathrm{O} 2$. The function of Catalase (CAT) is the catalytic decomposition of $\mathrm{H} 2 \mathrm{O}$ to $\mathrm{H} 2 \mathrm{O}$ and $\mathrm{O} 2, \mathrm{H} 2 \mathrm{O} 2$ prevent the $\mathrm{H} 2 \mathrm{O} 2$ from reacting with $\mathrm{O} 2$, thus generating the harmful hydroxyl radicals. GSH - Px (glutathione peroxidase) is also an important antioxidant enzyme in the body, it is not only has a strong cleaning power over the $\cdot \mathrm{OH}$-induced reactive oxygen species and lipid hydroperoxide, but also works as a specific catalytic in reduction glutathione reduction reaction for H2O2[4] . Body SOD, CAT and GSH - Px synergy to form a mutual defense groups to defense attacks from free radicals, preventing lipid peroxidation, protecting the body from sports injury[5]. MDA is the most important lipid peroxides generated from lipid peroxidation and also a sensitive indicator of measuring the level of free radical metabolism. After taking jujube polysaccharide, myocardial results of SOD, CAT and MDA GSH2Px shows that jujube polysaccharide can increase the activity of endogenous antioxidant enzyme, remove the effect of excess free radicals produced during the metabolism, which is of great importance in preventing lipid peroxidation and myocardial protection against sports injuries. The mechanism may be: 1) jujube polysaccharide can improve myocardial energy metabolism, enhancing glycolysis and aerobic decomposition, reducing the generation and accumulation of free radicals. 2) jujube polysaccharide can increase the myocardial tissue antioxidant enzyme activity and defense the free radical attack and biofilm inhibition of lipid peroxidation; 3) by blocking Ca2+,jujube polysaccharide can alleviate calcium overload, inhibit the release of lysosomal enzymes and a series of abnormal reaction caused by a high concentration of intracellular calcium ion[6].

Under normal resting conditions, serum enzyme activity remained relatively stable. When sports injuries and lesions occur in the tissues and organs (such as myocardial infarction, liver cell necrosis, malignant tumor, myositis, etc.), serum activity will subsequently change and the degree of change varies due to disease status, exercise load size and individual differences. Aspartate transaminase (GOT) is catalyzed oxaloacetate and glutamate deaminase enzymes, mainly distributed in the cytoplasm and the mitochondrial matrix, especially in heart, kidney, skeletal muscle. Serum GOT activity will directly indicate the extent of myocardial cell injury, which is also an important indicator for the clinical diagnosis of myocardial infarction, myocarditis[7]. The experimental results show that compared with the quiet group, serum GOT activity in the exercise group was significantly increased, indicating radicals produced by high-intensity endurance training can lead to accumulation of myocardial cell damage.

\section{Summary}

The result should that jujube polysaccharide can increase the activity of endogenous antioxidant enzyme, remove the effect of excess free radicals produced during the metabolism, which is of great importance in preventing lipid peroxidation and myocardial protection against sports injuries.

The fact that the GOT activity in group that has taken jujube polysaccharide was lower than the exercise control group can further explain that jujube polysaccharide has a protective effect on myocardial tissue.

\section{References}

[1] HOU Tao, Research Progress in Extraction and Biological Activities of Jujube Polysaccharides, The Beverage Industry. 16(2013)214-216. 
[2] Jin-wei Li, Shao-dong Ding, Xiao-lin Ding, Optimization of the ultrasonically assisted extraction of polysaccharides from Zizyphus jujubacv.Jinsixiaozao, Journal of Food Engineering. 166(2007)176-183.

[3] Dalsgaard MK, Fuelling cerebral activity in exercising man, Journal of Cerebral Blood Flow and Metabolism. 6(2006)731-750.

[4] Garden DL, Granger DN. Pathophysiology of ischemia-reperfusion injury, I Pathol. 190(200)255-266.

[5] Dalsgaard MK, Quistorff B, Danielsen ER, A reduced cerebral metabolic ratio in exercise reflects metabolism and not accumulation of lactate within the human brain, The Journal of Physiology. 2(2004)571-578.

[6] Bell RM, Maddock HL, Yellon DM, The cardioprotective and mitochondrial depolarising properties of exogenous nitric oxide in mouse heart, Cardiovasc Res. 57(2003)405-415.

[7] Kemppainen J, Aaho S, Fujimoto T, High intensity exercise decreases global brain glucose uptake in humans, The Journal of Physiology. 151(2005):323-332. 